\title{
Multilocus sequence typing of a dairy-associated Leuconostoc mesenteroides population reveals clonal structure with intragenic homologous recombination
}

\author{
Wenyi Zhang, Wenjun Liu, Yuqing Song, Haiyan Xu, Bilige Menghe, Heping Zhang, and Zhihong Sun ${ }^{1}$ \\ Key Laboratory of Dairy Biotechnology and Engineering, Education Ministry of P. R. China, Inner Mongolia Agricultural University, Hohhot, \\ Inner Mongolia 010018, P. R. China
}

\begin{abstract}
Leuconostoc mesenteroides strains play an important role in food fermentation. In this study, 136 strains from different dairy products in China and Mongolia were examined by multilocus sequence typing of 9 housekeeping genes. In total, 82 polymorphic sites were detected among the 9 loci. The number of polymorphic nucleotide sites varied between 4 (dnaA) and 18 (uvrC), whereas the nucleotide diversity per site among the 9 genes varied from 0.00379 in $d n a A$ to 0.01195 in uvrC, suggesting a relatively low level of sequence diversity. For the recombination measurement, incongruence in the trees based on a single gene and concatenated sequences of all sequencing types were observed, indicative of extensive intragenic homologous recombination. The overall relatedness built by minimum spanning trees showed no clear relationship between the clonal complexes and either isolation source or sampling location of the strains. Our study presents, for the first time, the population structure of Leuc. mesenteroides strains of dairy origin.
\end{abstract}

Key words: Leuconostoc mesenteroides, multilocus sequence typing, homologous recombination, clonal structure

\section{INTRODUCTION}

Leuconostoc mesenteroides are gram-positive, nonmotile, non-spore-forming facultative anaerobes, which require complex stimulation factors during growth. Their presence is detected in various food-related ecological niches, including beverages, meat products, milk products, and some plant materials (Hemme and Foucaud-Scheunemann, 2004). The first description of Leuconostoc mesenteroides was by Van Tieghem in 1878

Received December 11, 2014.

Accepted December 31, 2014

${ }^{1}$ Corresponding author: sunzhihong78@163.com and it was later proposed as the type strain (Garvie, 1979). In 1983, Leuconostoc dextranicum and Leuconostoc cremoris were reclassified as subspecies of Leuc. mesenteroides because of the common properties they shared with Leuc. mesenteroides (Garvie, 1983). These 3 organisms were reported to have similar lactate dehydrogenases and glucose-6-phosphate dehydrogenases (Garvie, 1983). When DNA-DNA hybridization was performed, the 3 strains showed a high degree of relatedness: Leuc. mesenteroides had $110 \pm 9.3 \%$ and 66 $\pm 10.3 \%$ homology with Leuc. dextranicum and Leuc. cremoris, respectively (Garvie, 1976). In a more recent study, a novel subspecies, Leuc. mesenteroides ssp. suionicum, was proposed based on the data obtained using a polyphasic approach (Gu et al., 2012).

Taxonomic studies of Leuc. mesenteroides initially focused mostly on physiological tests. Unfortunately, concerns have been raised about the phenotypic markers used during the process regarding their instability and irreproducibility, especially when applied to discriminate closely related species (Thunell, 1995). In fact, Barrangou et al. (2002) pointed out that the historical failure to differentiate Leuconostoc fallax from Leuc. mesenteroides was due to a lack of proper molecular identification methods. To address these serious concerns, molecular methods such as random amplified polymorphic DNA analysis, DNA fingerprinting and restriction of ribosomal DNA were developed and implemented (Villani et al., 1997; Pérez et al., 2002), which proved effective for discriminating Leuc. mesenteroides at the species level. However, continuous improvement and standardization of the methods for taxonomic study is still necessary because comparison of results between different laboratories remains challenging (Maiden, 2006).

Multilocus sequence typing (MLST), a new generic typing method, has been principally used in studies of Lactobacillus within the community of lactic acid bacteria (LAB; Tanigawa and Watanabe, 2011; BuhnikRosenblau et al., 2012; Chaillou et al., 2013). Herein, we used MLST to probe the evolution and population 
structure of 136 Leuc. mesenteroides isolates from different dairy products in China and Mongolia. To our knowledge, this is the first MLST scheme for Leuc. mesenteroides. We believe that the development and application of MLST for this species will promote further use of the strains in food fermentation.

\section{MATERIALS AND METHODS}

\section{Bacterial Isolates and DNA Extraction}

One hundred thirty-six Leuc. mesenteroides strains were obtained from the Collection Centre of Lactic Acid Bacteria at Inner Mongolia Agriculture University of China (Hohhot). These strains were isolated from dairy products (whey, yogurt, yak milk, and qula, a traditional cheese) in 3 provinces of China and 5 provinces of Mongolia (Supplemental Table S1; http://dx.doi. org/10.3168/jds.2014-9227). The strains were grown in $5.0 \mathrm{~mL}$ of M17 broth (CM0817B, Oxoid, Wesel, Germany) at $30^{\circ} \mathrm{C}$ for 18 to $22 \mathrm{~h}$. Total genomic DNA was extracted from cultures as described by $\mathrm{Yu}$ et al. (2012). For further use, purified DNA was diluted to a final concentration of $100 \mathrm{ng} / \mu \mathrm{L}$.

\section{Selection of MLST Loci}

With reference to the genome sequence of Leuc. mesenteroides ATCC8293 (GenBank accession number: CP000414.1), 9 housekeeping genes (dnaA, groEL, murC, murE, pepN, pheS, pyrG, rpoB, and uvrC) were selected for MLST analysis. These genes are evenly separated across the entire genome, conserved, and well characterized (Cai et al., 2007; Bilhère et al., 2009). The primers for these 9 genes were designed using the Primer Premier 5.0 program (Premier Biosoft International, Palo Alto, CA) and are listed in Table 1.

\section{PCR Amplification and DNA Sequencing}

The PCR amplifications of MLST loci were carried out in an automatic thermal cycler (PTC-200, MJ Research, Waltham, MA). The thermal cycling conditions were as follows: $94^{\circ} \mathrm{C}$ for $5 \mathrm{~min}$; 30 cycles of $94^{\circ} \mathrm{C}$ for $1 \mathrm{~min}$, annealing temperature of each locus for $1 \mathrm{~min}$, and $72^{\circ} \mathrm{C}$ for $2 \mathrm{~min}$; and a final elongation step of $72^{\circ} \mathrm{C}$ for $8 \mathrm{~min}$. The PCR mixture $(50 \mu \mathrm{L})$ for each target contained $150 \mathrm{ng}$ of genomic DNA, $10 \mathrm{~m} M$ of each deoxynucleotide triphosphates (dNTP), $10 \mathrm{pmol}$ of each primer, and $2.5 \mathrm{U}$ of Taq polymerase in $1 \times \mathrm{PCR}$ buffer (with $\mathrm{Mg}^{2+}$ ). The PCR products were first analyzed by electrophoresis in a $1.2 \%$ agarose gel and were then sequenced by Shanghai Majorbio Bio-Pharm Technology Corporation (Shanghai, China). The same primers

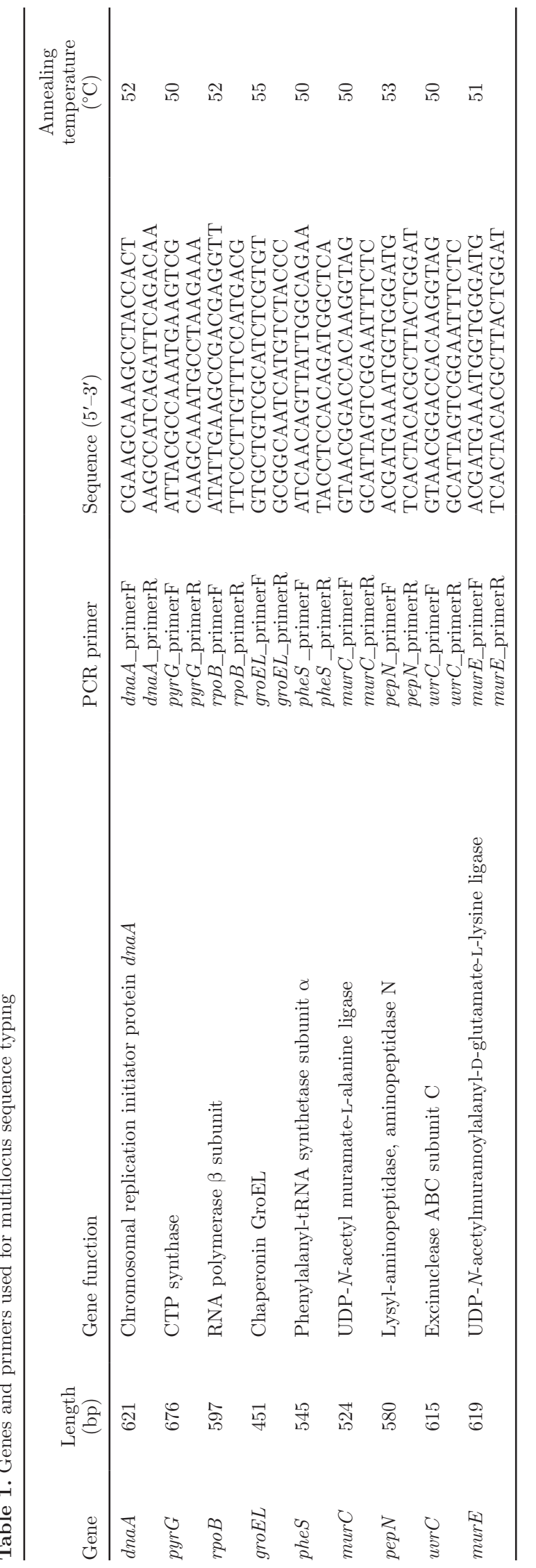

Journal of Dairy Science Vol. 98 No. 4, 2015 
from the PCR amplifications were used for sequencing on both DNA strands.

\section{Data Analysis}

For MLST analysis, forward and reverse sequences were trimmed, aligned, and analyzed using MEGA 5.0 (http://www.megasoftware.net/). Definition of alleles using a nonredundant data set and minimum spanning tree analysis were based on BioNumerics 6.6 (Applied-Maths, Sint Maartens-Latem, Belgium). Allelic sequences having more than one nucleotide difference were assigned different arbitrary numbers. For each of the 9 MLST loci or alleles, a unique nucleotide sequence was assigned. The sequence types (ST) were defined by unique allelic profiles (consisting of the allele numbers at each of the 9 MLST loci). The same ST was used for several strains if they have common allelic profiles. Groups of isolates with closely related allelic profiles were regarded as clonal complexes $(\mathbf{C C})$. The ST were grouped into CC using the eBURST (Based Upon Related Sequence Types) program (http:// eburst.mlst.net/; Feil et al., 2004) and were identified in the BioNumerics program. In our study, an ST was defined as a subgroup founder that had $>4$ single locus variants (SLV).

Descriptive evolutionary analyses such as $\mathrm{G}+\mathrm{C}$ content $(\mathrm{mol} \%) ; d_{N} / d_{S}$ ratio, where $d_{N}$ is the number of nonsynonymous substitutions per nonsynonymous site, and $d_{S}$ is the number of synonymous substitutions per synonymous site; nucleotide diversity $(\pi)$ per site; number of polymorphic sites; Tajima's D test, and Fu and Li's D and F tests (the values from all 3 tests that did not significantly deviate from zero indicate potential negative selection in the loci) were calculated using DnaSP 5.0 (Librado and Rozas, 2009). With the program LIAN3.5, linkage equilibrium between alleles at the 9 housekeeping genes was assessed, based on the standardized index of association $\left(\boldsymbol{I}_{\boldsymbol{A}}{ }^{S}\right.$; Haubold and Hudson, 2000). To evaluate the degree of tree-like structure for alleles of each locus and all ST, the split decomposition method was performed with SplitsTree 4.0 (Huson and Bryant, 2006). Phylogenetic trees were constructed by the neighbor-joining method using MEGA 5.0 software package (http://www.megasoftware.net/), with 1,000 replicates in bootstrap analysis.

\section{Nucleotide Sequence Accession Numbers}

Allele sequences of the 9 MLST loci have been deposited in the GenBank under accession numbers KP035238 to KP036325.

\section{RESULTS}

\section{PCR Amplification and Sequencing and Definition of ST}

Nine genes fulfilling the criteria of MLST analysis were amplified and sequenced for the present study. The length of the genes ranged from 321 to $496 \mathrm{bp}$. Sixty-eight different ST were identified among 136 Leuc. mesenteroides strains (Supplemental Table S2; http://dx.doi.org/10.3168/jds.2014-9227). Of these, ST-14 was the most represented and was composed of 13 strains, followed by ST-29 (10 strains), ST-11 (9 strains), ST-15 (6 strains), ST-55 (6 strains), ST-9 (4 strains), and other ST with fewer than 4 strains.

\section{Allelic Variation at the MLST Loci}

The nucleotide diversity at each locus of the strains is described in Table 2. Among the 9 loci, 82 polymorphic sites were detected in the 136 isolates of Leuc. mesenteroides. The number of polymorphic nucleotide sites varied between $4($ dnaA) and 18 (uvrC). The mean $\mathrm{G}+\mathrm{C}$ content (mol \%) observed for different gene fragments was $40.5 \%$, within a range from 40.48 to $40.67 \%$. The nucleotide diversity per site $(\pi)$ among the 9 genes varied from 0.00379 in $d n a A$ to 0.01195 in $u v r C$. The $d_{N} / d_{S}$ ratios of 9 loci ranged from 0.0219 to 2.4379 . Except for that of murC, most $d_{N} / d_{S}$ ratios were $<1$ (Table 2), suggesting that no strong positive selective pressure was present. Accordingly, the values from Tajima's D test, and Fu and Li's D and F tests did not show a significant deviation from 0 (Table 2).

\section{Detection for Recombination}

Evidence for recombination was determined using 2 different approaches. The result based on $I_{A}{ }^{S}$ analysis revealed a value of 0.1661 based on the 9 chosen loci. The observed variance was greater than the maximum variance obtained after 1,000 trials $(P=0.000)$; therefore, the population was in complete linkage equilibrium. This finding also shows that all the studied isolates together form an underlying clonal population structure.

For further measurement of intragenic recombination, split-decomposition analysis was performed on each locus separately and on the concatenated sequences of all ST. As shown in the split graphs (Figure 1), 7 loci (groEL, murE, pepN, pheS, pyrG, rpoB, and uvrC) showed classical tree-like structures, whereas a possible parallelogram structure was detected for loci dnaA and murC. In contrast, the concatenated sequence of 
Table 2. Descriptive analysis of genetic variability at Leuconostoc mesenteroides loci

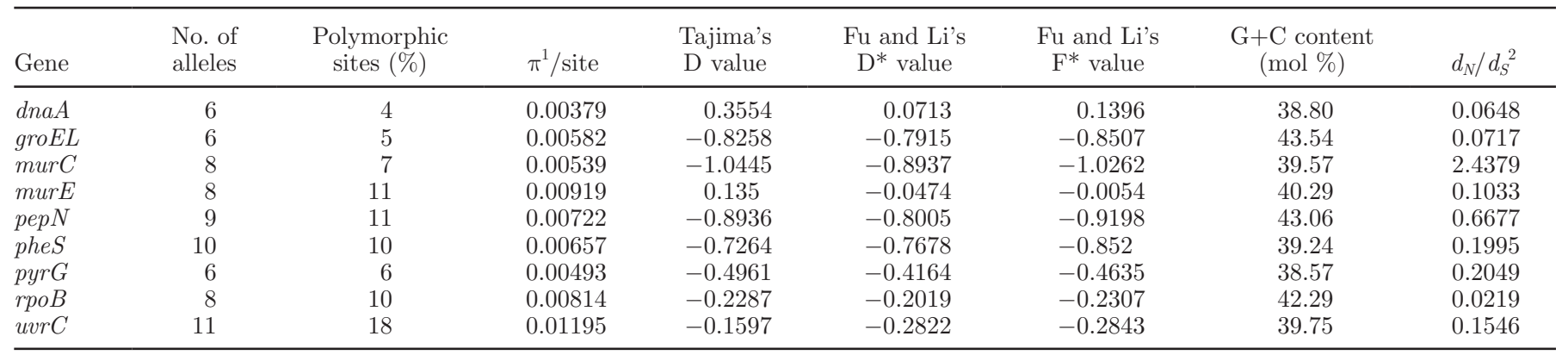

${ }^{1}$ Mean pairwise nucleotide difference per site.

${ }^{2} d_{N} / d_{S}$ represents the ratio of nonsynonymous to synonymous substitutions.

the 9 genes displayed a typical extensive network-like structure (Figure 2), suggesting that most sections among the 9 genes had pervasive and associative recombination (Phi test, $P=1.434 \mathrm{E}-6$ ). No apparent difference was observed on the overall topology if data from either $d n a A$ or $\operatorname{mur} C$ were removed before tree construction (Supplemental Figures S1 and S2; http:// dx.doi.org/10.3168/jds.2014-9227).

\section{Population Structure of Leuc. mesenteroides Strains}

A snapshot of the population structure was obtained by eBURST analysis, with a stringent group definition of 7 of 9 shared alleles. As showed in Figure 3, $8 \mathrm{CC}$ (CC1 to CC8) and 16 singletons were observed for the $52 \mathrm{ST}$. The number of the strains contained in these $\mathrm{CC}$ accounted for $70.59 \%$ of the strain collection.

Among the $8 \mathrm{CC}, \mathrm{CC} 1$ was the largest and comprised 16 linked ST; namely, ST24, ST15, ST55, ST68, ST62, ST54, ST57, ST60, ST65, ST38, ST37, ST3, ST5, ST6, ST49, and ST66. These ST included 11 strains isolated from Inner Mongolia, 8 isolates from Gansu province and 4 isolates from Sichuan province in China. Clonal complex 2, representing $7 \mathrm{ST}$, consisted of 12 strains, with 5 of them isolated from Inner Mongolia, 4 from Gansu province, and 3 from Sichuan province. Clonal complex 3 contained 17 strains; most were isolated from Sichuan province (10 strains). Clonal complex 4 had 6 strains, 4 from Inner Mongolia, 1 from Sichuan province, and 1 from Mongolia. Clonal complex 5 had 21 stains, most of which were isolated from Inner Mongolia (17 strains), 2 from Sichuan province, and 2 from Gansu province. The rest of the CC were small clusters that had fewer than $5 \mathrm{ST}$ with limited strains isolated from one geographical location. Moreover, CC1, which had $12 \mathrm{SLV}$, appeared to be more complicated than any other CC. We therefore assigned it as the primary founder of the CC.
In the minimum spanning tree featured with isolates from different regions (Figure 4), we found 4 main branches. Interestingly, strains isolated from Mongolia and Inner Mongolia showed closer relationships than strains originating from other regions. Branch 1 corresponded to CC1, which contained strains isolated from Inner Mongolia, Gansu, and Sichuan. Branch 2 was nearly identical to CC5, having most strains isolated from Inner Mongolia. Branch 3 consisted of the strains from CC2, which were isolated from Gansu, Sichuan, and Inner Mongolia. Branch 4 included CC7, CC3, and CC6, with most strains from Gansu province, Inner Mongolia, and Mongolia. When the minimum spanning tree was constructed based on the dairy sources of the isolates (Supplemental Figure S3; http://dx.doi. org/10.3168/jds.2014-9227), no clear relationship was seen between the clusters and the isolation sources, even considering the 2 main sources that included most strains.

A neighbor-joining tree was constructed using the concatenated sequences of the 9 genes from the 136 Leuc. mesenteroides strains (Supplemental Figure S4; http://dx.doi.org/10.3168/jds.2014-9227). However, no major clusters were identified with acceptable bootstrap values. Most branches of the tree were poorly supported by bootstrap values of $<70 \%$, which means that the corresponding clades were not likely to be real (Hillis and Bull, 2004).

\section{DISCUSSION}

Multilocus sequence typing is a powerful technique to characterize bacteria at the molecular level. Because it is a sequence-based method, comparisons of the experimental results from different labs become more convenient. Moreover, data can be retrieved through open databases via the Internet. In the present study, MLST was used to explore the population structure 


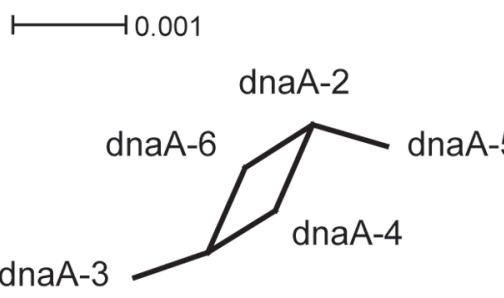

dnaA-1
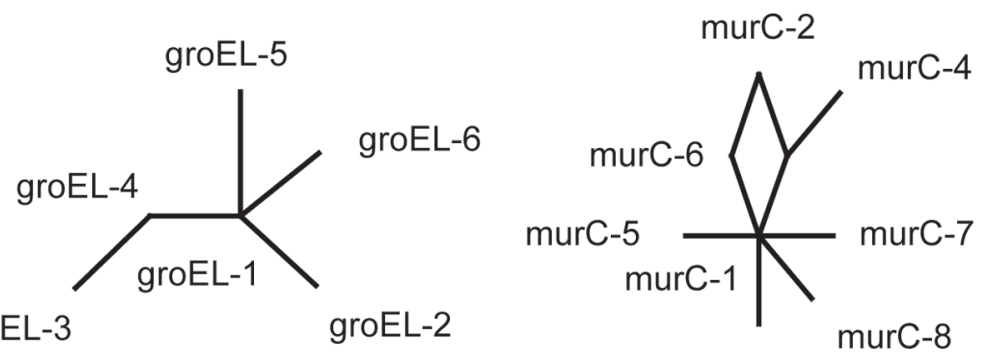

murC-3
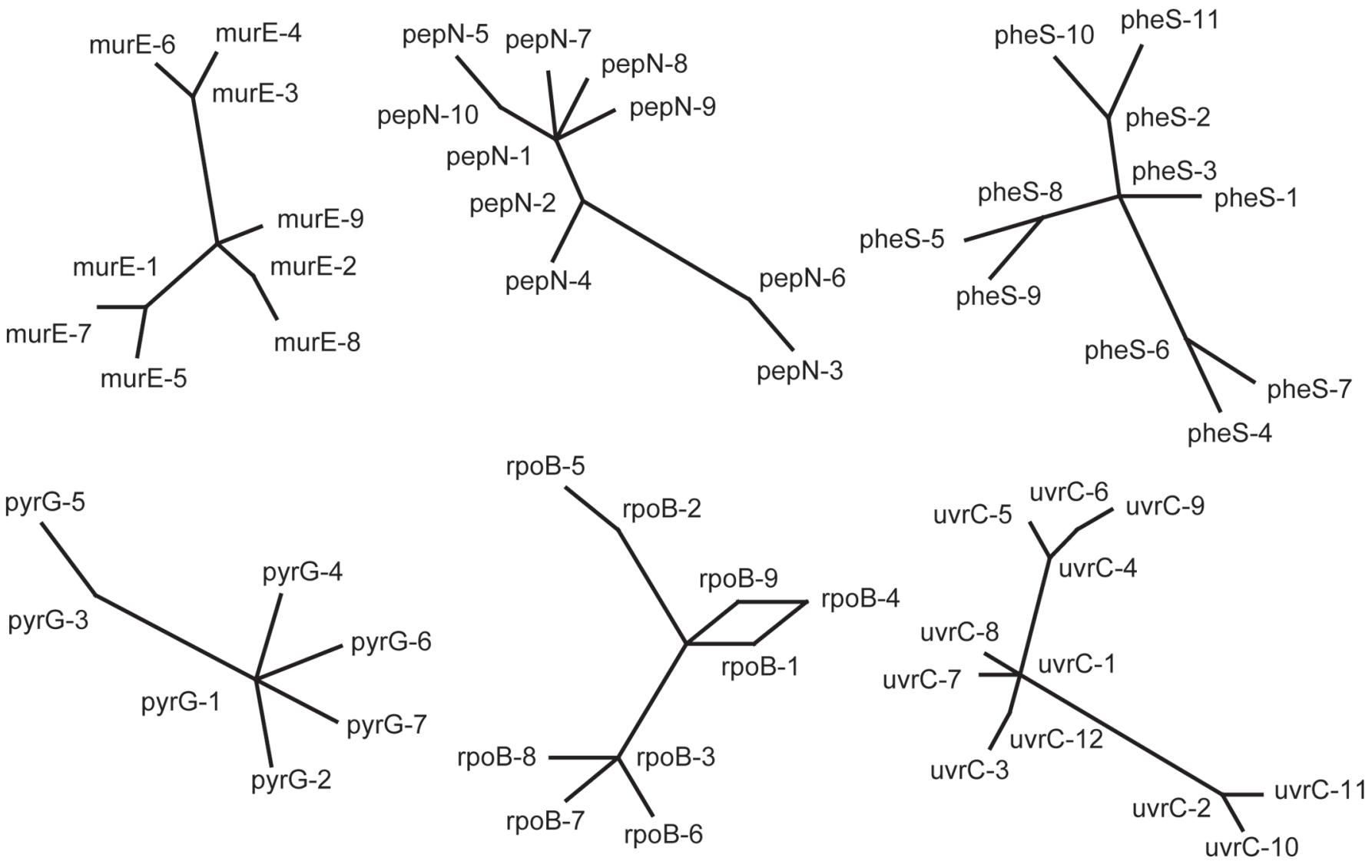

Figure 1. Split-decomposition analysis of alleles for 9 individual multilocus sequence typing (MLST) loci (dnaA, groEL, murC, murE, pepN, pheS, pyrG, rpoB, and uvrC) of 136 Leuconostoc mesenteroides strains.

and evolution of 136 Leuc. mesenteroides isolates from different ecological and geographical origins, which may provide better information concerning their biological properties.

To initiate analysis, the sequence diversity of the 9 housekeeping genes was calculated. This step was carried out to measure whether these selected loci had sufficient typing discrimination. The number of alleles in these gene loci ranged from 6 to 11. This observation corroborates the previously described genetic heterogeneity of Leuconostoc lactis (Dan et al., 2014). Compared with nucleotide sequence diversities reported in Lactobacillus, such as Lactobacillus sakei and Lactobacillus casei (Cai et al., 2007; Chaillou et al., 2013), Leuc. mesenteroides housekeeping genes have similar but lower $\pi$ values in general, reflecting that these genes are conserved. Consistently, the 9 loci were polymorphic, and 2 were relatively less diverse (polymorphic sites $\leq 5$ ) among the examined isolates.

By analyzing the synonymous and nonsynonymous changes in the allele sequences, a small $d_{N} / d_{S}$ ratio of $\ll 1$ was obtained, which implies that the selected genes were subjected to negative selection. This trend of divergences in the selected genes made them suitable 


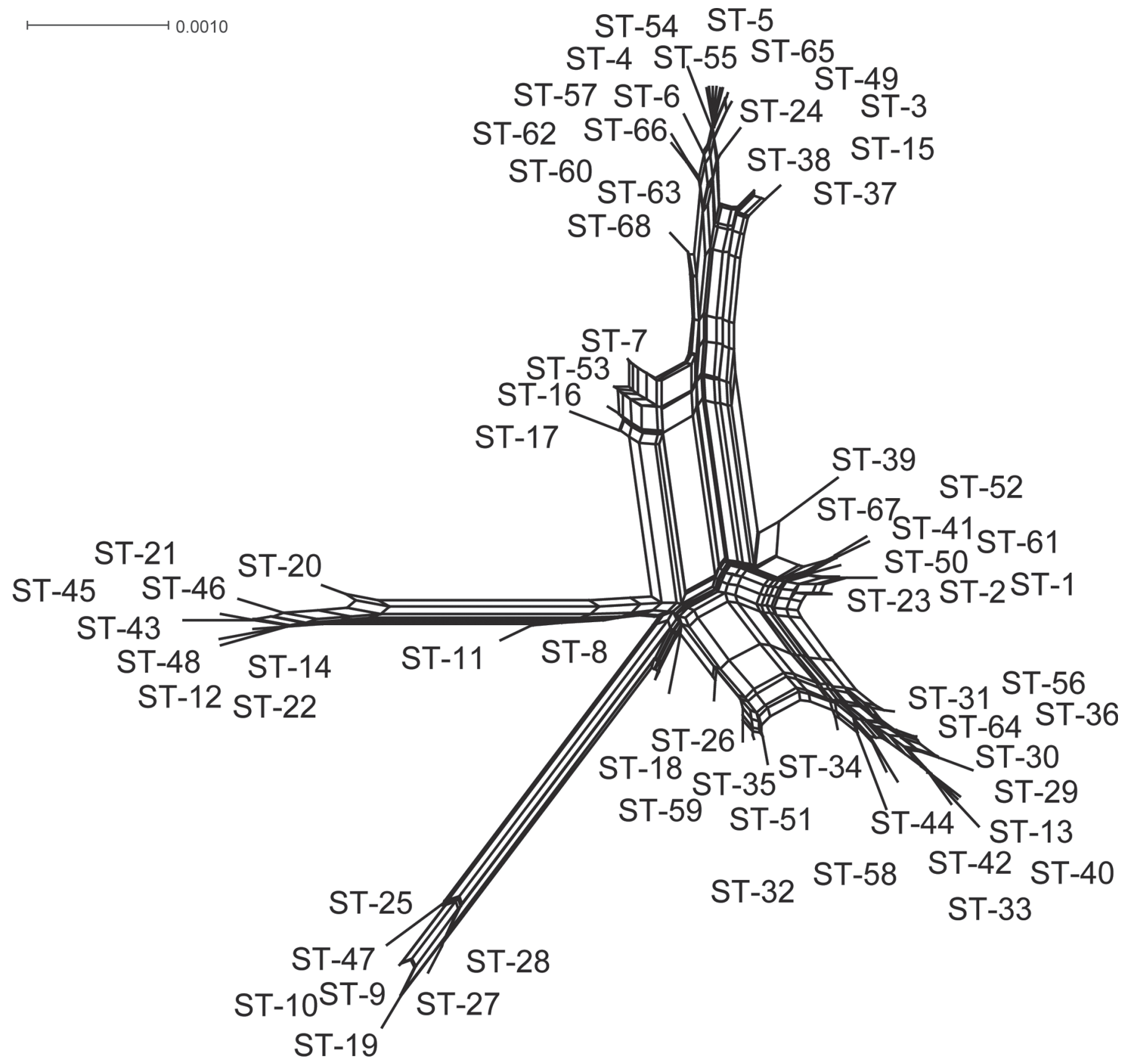

Figure 2. Combined split-decomposition analysis of alleles for the 9 sequence type (ST) loci (dnaA, groEL, murC, murE, pepN, pheS, pyrG, rpoB, and uvrC) of the 136 Leuconostoc mesenteroides strains.

for analysis by MLST. One exception was found with gene $\operatorname{mur} C$, which had a $d_{N} / d_{S}$ ratio $>1$, which is not typically observed for housekeeping genes. Nevertheless, this gene has been used in the molecular typing of Lactococcus lactis, which showed abundant polymorphisms (Xu et al., 2014). However, after sequencing the fragment 194 bp distant from the $5^{\prime}$ end of murC, only 7 polymorphic sites, equivalent to $1.33 \%$ of all sites, were observed. The apparent low level of polymorphisms led us to believe that this gene was not genetically as diverse as those of other LAB species. Similar observations were reported for the polymorphism of gtfB gene in Streptococcus mutans isolated from 5-yr-old children and their mothers (Toi et al., 2005).

Several studies have questioned the discriminatory power of single or multiple gene-based phylogeny (Simpson et al., 2004). Because of potential horizontal gene transfer and extensive homologous recombination, 


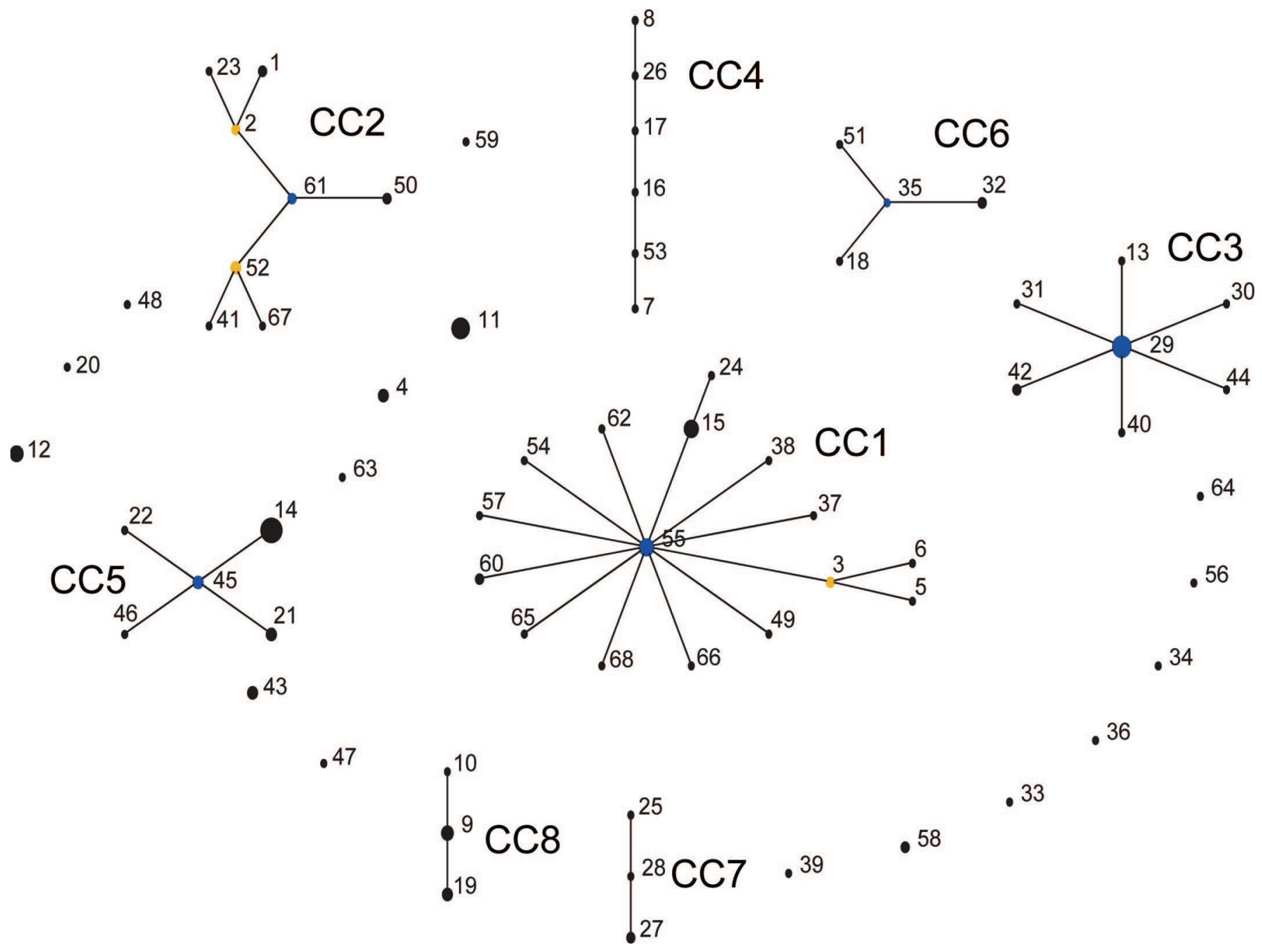

Figure 3. Population structure of 136 Leuconostoc mesenteroides isolates by eBURST analysis (Based Upon Related Sequence Types; http:// eburst.mlst.net/). $\mathrm{CC}=$ clonal complex.

phylogenetic trees built based on a limited number of genes could result in misleading inferences. For example, homologous recombination can occur even in the highly conserved rRNA sequences, which reduces the reliability of using the rRNA sequences as traditional markers in phylogenetic analysis (Schouls et al., 2003). The housekeeping genes used here for MLST analysis had similar $\mathrm{G}+\mathrm{C}$ contents compared with the overall genome of the type strain Leuc. mesenteroides ATCC8293, suggesting that they originated from the bacterial genome rather than being acquired via horizontal gene transfer events. For the recombination measurement, incongruence in the trees based on single gene and concatenated sequences of all ST was observed, indicating extensive intragenic recombination. Accordingly, the phylogeny established using the neighbor-joining method failed to show reliable topology with poorly supported bootstrapping values. This fact implied that the neighborjoining method was unsuitable for analyzing MLST data of the current Leuc. mesenteroides strains. In a previous study, Diancourt et al. (2007) pointed out that approaches such as eBURST and minimum spanning that are less sensitive to the effects of recombination might be preferable.

As indicated by the large number of the strains involved in CC, our study presented a clonal structure of Leuc. mesenteroides strains isolated from dairyassociated niches. One interesting application of the MLST technique is identification of associations between strains and their origin. For example, Cai et al. (2007) reported the first MLST study of LAB, in which a collection of 40 Lactobacillus casei strains were 


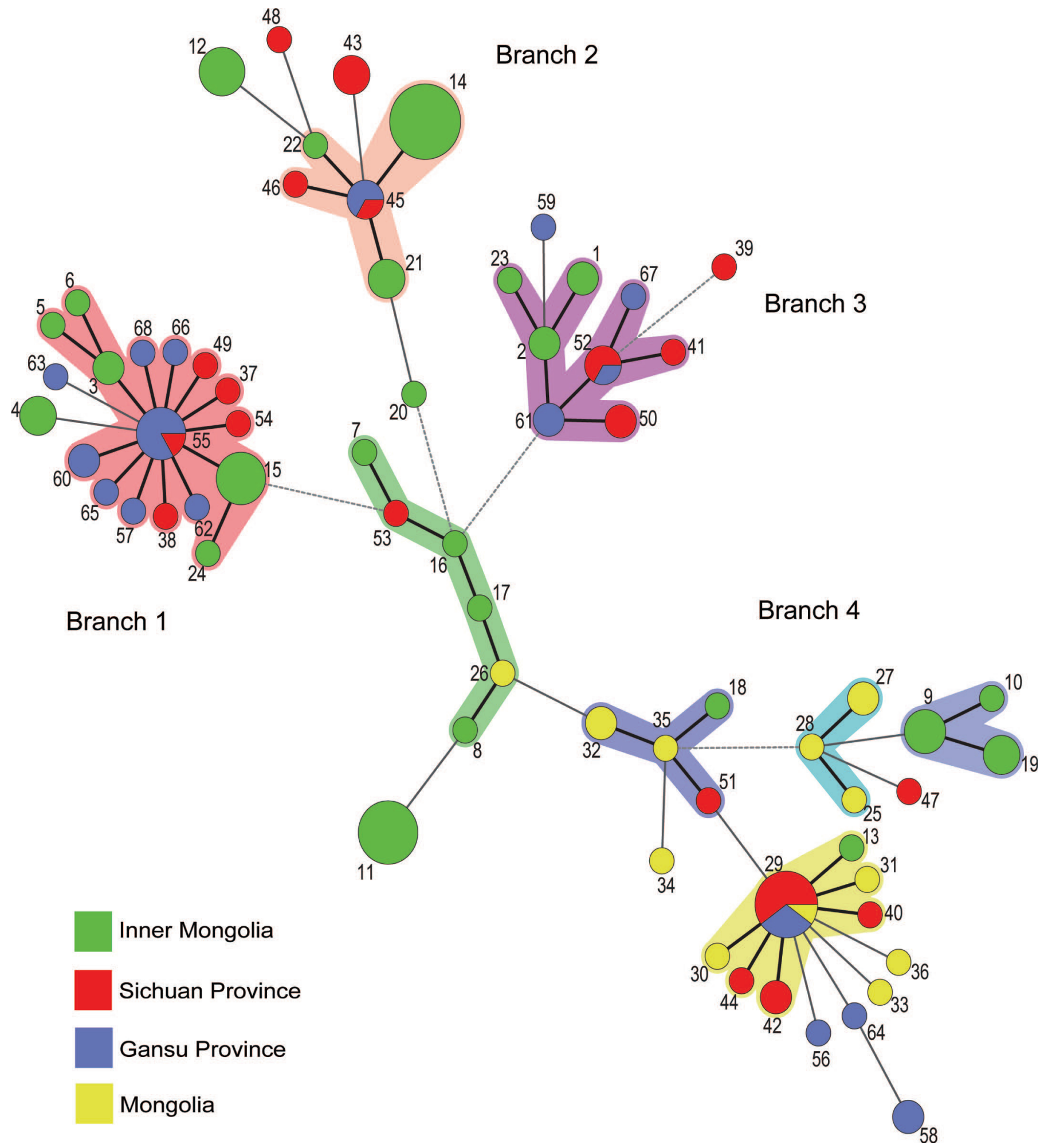

Figure 4. Minimum-spanning tree analysis of the 136 Leuconostoc mesenteroides strains from different regions. Each circle corresponds to a sequence type (ST), and the circle size denotes the number of strains sharing the same ST. Numbers between the circles indicate the number of allelic differences between the profiles. The strength of the link (solid, bold, or dashed) is related to the genetic similarity (number of common alleles) between profiles. 
grouped into a cheese cluster, a silage cluster, and a mixed cluster primarily consisting of the human gastrointestinal tract and cheese isolates. Some correlation was observed between the minimum spanning tree and the pulsed-field gel electrophoresis tree, suggesting high specificity of the individual ecological niches. However, in this study, no strong relationship between the CC and the origins of the strains included in them could be identified, based either on the dairy source or on sampling location. Often, only some strains of the same source or location were clustered together, whereas the rest were dispersed across other clusters. One possible explanation for the weak correlation was the open market for international and regional trading of dairy products, which might have increased the occurrence of the genetic exchange between strains. The absence of such a relationship was reported in typing analyses of Lactobacillus delbrueckii, Lactococcus lactis, and Lactobacillus sakei (Passerini et al., 2010; Tanigawa and Watanabe, 2011; Chaillou et al., 2013).

\section{CONCLUSIONS}

Leuconostoc mesenteroides is important in food fermentation, and the species can be detected in a variety of fermented food-related ecological niches. We developed an MLST scheme for its typing analysis and investigated the population biology within a set of 136 strains. Our results showed that intragenic homologous recombination plays a key role in Leuc. mesenteroides diversity. However, the strains studied here were entirely of dairy origin, which may introduce bias in representing the genetic heterogeneity of this species, because dairy strains may display lower diversity compared with nondairy strains. It would be interesting to analyze the population structure of Leuc. mesenteroides from various niches by combined use of MLST and whole-genome resequencing techniques.

\section{ACKNOWLEDGMENTS}

This research was supported by the National Natural Science Foundation of China (Beijing; grant no. 31201396), Hi-Tech Research and Development Program of China (Beijing; 863 Planning, grant nos. 2011AA100901 and 2011AA100902), International S\&T Cooperation Program of China (Beijing; ISTCP, grant no. 2014DFR31150), the China Agriculture Research System (Beijing; grant no. CARS-37), and the Excellent Young Scientist Foundation of Inner Mongolia Agricultural University of China (Huhhot; grant no. 2014XYQ-16).

\section{REFERENCES}

Barrangou, R., S. S. Yoon, F. Breidt Jr., H. P. Fleming, and T. R. Klaenhammer. 2002. Identification and characterization of Leuconostoc fallax strains isolated from an industrial sauerkraut fermentation. Appl. Environ. Microbiol. 68:2877-2884.

Bilhère, E., P. M. Lucas, O. Claisse, and A. Lonvaud-Funel. 2009. Multilocus sequence typing of Oenococcus oeni: Detection of two subpopulations shaped by intergenic recombination. Appl. Environ. Microbiol. 75:1291-1300.

Buhnik-Rosenblau, K., V. Matsko-Efimov, M. Jung, H. Shin, Y. Danin-Poleg, and Y. Kashi. 2012. Indication for co-evolution of Lactobacillus johnsonii with its hosts. BMC Microbiol. 12:149.

Cai, H., B. T. Rodriguez, W. Zhang, J. R. Broadbent, and J. L. Steele. 2007. Genotypic and phenotypic characterization of Lactobacillus casei strains isolated from different ecological niches suggests frequent recombination and niche specificity. Microbiology 153:2655-2665.

Chaillou, S., I. Lucquin, A. Najjari, M. Zagorec, and M. C. Champomier-Verges. 2013. Population genetics of Lactobacillus sakei reveals three lineages with distinct evolutionary histories. PLOS ONE 8:e73253.

Dan, T., W. Liu, Z. Sun, Q. Lv, H. Xu, Y. Song, and H. Zhang. 2014. A novel multi-locus sequence typing (MLST) protocol for Leuconostoc lactis isolates from traditional dairy products in China and Mongolia. BMC Microbiol. 14:150.

Diancourt, L., V. Passet, C. Chervaux, P. Garault, T. Smokvina, and S. Brisse. 2007. Multilocus sequence typing of Lactobacillus casei reveals a clonal population structure with low levels of homologous recombination. Appl. Environ. Microbiol. 73:6601-6611.

Feil, E. J., B. C. Li, D. M. Aanensen, W. P. Hanage, and B. G. Spratt. 2004. eBURST: Inferring patterns of evolutionary descent among clusters of related bacterial genotypes from multilocus sequence typing data. J. Bacteriol. 186:1518-1530.

Garvie, E. 1979. Proposal of neotype strains for Leuconostoc mesenteroides (Tsenkovskii) van Tieghem, Leuconostoc dextranicum (Beijerinck) Hucker and Pederson, and Leuconostoc cremoris (Knudsen and Sorensen) Garvie. Int. J. Syst. Bacteriol. 29:149-151.

Garvie, E. I. 1976. Hybridization between the deoxyribonucleic acids of some strains of heterofermentative lactic acid bacteria. Int. J. Syst. Bacteriol. 26:116-122.

Garvie, E. I. 1983. Leuconostoc mesenteroides ssp. cremoris (Knudsen and Sorensen) comb. nov. and Leuconostoc mesenteroides ssp. dextranicum (Beijerinck) comb. nov. Int. J. Syst. Bacteriol. 33:118119.

Gu, C. T., F. Wang, C. Y. Li, F. Liu, and G. C. Huo. 2012. Leuconostoc mesenteroides ssp. suionicum ssp. nov. Int. J. Syst. Evol. Microbiol. 62:1548-1551.

Haubold, B., and R. R. Hudson. 2000. LIAN 3.0: Detecting linkage disequilibrium in multilocus data. Linkage Analysis. Bioinformatics $16: 847-848$.

Hemme, D., and C. Foucaud-Scheunemann. 2004. Leuconostoc, characteristics, use in dairy technology and prospects in functional foods. Int. J. Dairy Technol. 14:467-494.

Hillis, D. M., and J. J. Bull. 2004. An empirical test of bootstrapping as a method for assessing confidence in phylogenetic analysis. Syst. Biol. 42:182-192.

Huson, D. H., and D. Bryant. 2006. Application of phylogenetic networks in evolutionary studies. Mol. Biol. Evol. 23:254-267.

Librado, P., and J. Rozas. 2009. DnaSP v5: A software for comprehensive analysis of DNA polymorphism data. Bioinformatics 25:1451-1452

Maiden, M. C. 2006. Multilocus sequence typing of bacteria. Annu. Rev. Microbiol. 60:561-588.

Passerini, D., C. Beltramo, M. Coddeville, Y. Quentin, P. Ritzenthaler, M. L. Daveran-Mingot, and P. Le Bourgeois. 2010. Genes but not genomes reveal bacterial domestication of Lactococcus lactis. PLOS ONE 5:e15306.

Pérez, G., E. Cardell, and V. Zarate. 2002. Random amplified polymorphic DNA analysis for differentiation of Leuconostoc mesen- 
teroides subspecies isolated from Tenerife cheese. Lett. Appl. Microbiol. 34:82-85.

Schouls, L. M., C. S. Schot, and J. A. Jacobs. 2003. Horizontal transfer of segments of the 16S rRNA genes between species of the Streptococcus anginosus group. J. Bacteriol. 185:7241-7246.

Simpson, P. J., R. P. Ross, G. F. Fitzgerald, and C. Stanton. 2004. Bifidobacterium psychraerophilum sp. nov. and Aeriscardovia aeriphila gen. nov., sp. nov., isolated from a porcine caecum. Int. J. Syst. Evol. Microbiol. 54:401-406.

Tanigawa, K., and K. Watanabe. 2011. Multilocus sequence typing reveals a novel subspeciation of Lactobacillus delbrueckii. Microbiology 157:727-738.

Thunell, R. K. 1995. Taxonomy of the Leuconostocs. J. Dairy Sci. 78:2514-2522.

Toi, C. S., P. Cleaton-Jones, and P. Fatti. 2005. Characterization of Streptococcus mutans diversity by determining restriction frag- ment-length polymorphisms of the gtfB gene of isolates from 5 -year-old children and their mothers. Antonie van Leeuwenhoek 88:75-85.

Villani, F., G. Moschetti, G. Blaiotta, and S. Coppola. 1997. Characterization of strains of Leuconostoc mesenteroides by analysis of soluble whole-cell protein pattern. DNA fingerprinting and restriction of ribosomal DNA. J. Appl. Microbiol. 82:578-588.

Xu, H., Z. Sun, W. Liu, J. Yu, Y. Song, Q. Lv, J. Zhang, Y. Shao, B. Menghe, and H. Zhang. 2014. Multilocus sequence typing of Lactococcus lactis from naturally fermented milk foods in ethnic minority areas of China. J. Dairy Sci. 97:2633-2645.

Yu, J., Z. Sun, W. Liu, Q. Bao, J. Zhang, and H. Zhang. 2012. Phylogenetic study of Lactobacillus acidophilus group, L. casei group and L. plantarum group based on partial hsp60, pheS and tuf gene sequences. Eur. Food Res. Technol. 234:927-934. 